\title{
Research on "half-and-half class" Teaching Mode for College Japanese Education in the Era of Information
}

\author{
Wanyu Li \\ (Foreign Language Department of Xinxiang College, Xingxiang, Henan 453003
}

\begin{abstract}
In the age of reform on education information, "half-and -half class" raised by the professor, Zhang Xuexing in Fudan University, is a new type of teaching model for current problems in classrooms of higher institutions whose core principles are a half of class hour given for teachers, and another half for students to interact with each other in manner of discussion. It not only maintains the advantages of traditional classes featuring teaching, but integrates with the merits of discussion classes with clear discussion structure "Liangkao Group". This thesis starts with current situations of college Japanese education, and combines practical discussion to explore the impacts of "half-and-half class" teaching model on education in the practical teaching of comprehensive Japanese majored by students in universities.
\end{abstract}

Keywords: Half-and -half class; Higher education; Comprehensive Japanese

\section{信息化时代下大学日语教学的 “对分课堂” 教学 \\ 模式研究}

李万豫

(新乡学院外国语学院 河南 新乡 453003)

摘要: 教育信息化改革的大背景下, 对分课堂是复旦大学张学新教授针对当前高校课堂教学中存在的问题 而提出的一种新的教学模式。该教学模式的核心理念是把一半的课堂时间分配给教师讲授, 另一半分配给学生 以讨论的形式进行交互式学习, 既保留了传统讲授式课堂的优势, 也融合了讨论式课堂的优点, 具有明确的“亮 考帮”讨论支架。本文从当前大学日语教学现状入手, 结合实际讨论, 在大学日语专业的综合日语的实际教学过 程中, 使用对分课堂的教学模式对教学的影响。

关键词：对分课堂；高校教育；综合日语

引言

近年来, 高校课堂缺勤率高, 学生玩手机、聊天、发呆, 对教学内容不感兴趣、不闻不问、不学不思 的情况相当普遍。教师的教学模式, 基本是老师怎么教, 学生怎么教学的传统模式上。往往教师认为课堂 讲过的知识, 学生就应该会, 学生具体能否灵活掌握和使用所学知识, 或者掌握到何种程度, 不得而知。 教师教学负担重、效果差, 心理压力大。针对当前高校课堂存在的这些问题, 结合讲授式课堂与讨论式课 堂的优点, 在大学日语专业的 “综合日语” 课程的教学当中, 尝试使用”对分课堂”的课堂教学改革新模式。

\section{1 “对分课堂” 的核心理念}

传统教学包括教师课堂讲授和学生课后学习两个相对分离的过程, 师生交互很少, 学生被动接受, 主 动性低, 难以培养思维能力和探索精神。讨论式教学通过课堂讨论引发学生主动学习的动力, 提升学习积 极性。然而课堂大部分时间用于讨论, 讲授过少, 不能充分发挥教师价值。 
对分课堂的核心理念是把一半课堂时间分配给教师进行讲授，另一半时间分配给学生以讨论的形式进 行交互式学习。在对分课堂上, 教师介绍基本框架、基本概念, 着重讲授重点、难点, 但并不穷尽教材内 容。学生通过教师讲授把握了章节的基本内容, 理解了重点、难点, 大大降低了课后的学习难度。课后学 习时, 学生可以根据自己的个人特点和具体情况, 以自己的节奏去完成内化吸收过程, 完成对教材内容更 为全面的学习与理解。内化吸收之后, 学生再回到课堂上, 分组讨论自己学过的内容, 然后与全班和教师 进行深入的互动交流。同一内容, 经过教师讲授、课后复习、分组讨论三个过程分阶段学习, 理解程度逐 步加深。本堂课讨论上堂课讲授的内容, 这样的 “隔堂讨论” 让学生有备而来, 显著提高了讨论质量, 活 跃了课堂气氛, 增强了学生的学习积极性和主动性。

这样, 在讲授和内化吸收之外, 突出了课堂讨论过程。实施中最关键的一点是把讲授和交互式学习在 时间上分隔开来 (或相隔一周), 让学生在这两个过程之间有充分的时间按自己的节奏进行个性化的内化 吸收。这样, 对分课堂把教学刻画为时间上清晰分离的三个过程, 分别为讲授 (Presentation)、内化吸 收 (Assimilation) 和讨论 (Discussion), 也可简称为 PAD 课堂。

目前对分课堂有隔堂对分、当堂对分及隔堂+当堂对分等三种操作模式。对分课堂体现了建构主义的 知识观、学习观及教学观, 具有可以提高学生学习自主性、关注不同层次的学生需求、操作简单易上手、 减轻教师工作量并提升职业价值感、真正做到教学相长并提升教师能力、增加师生及生生互动并最终提升 课堂教学效果和质量等优势。对分课堂注重教学流程的改革, 无需大量投入, 是一种经济、实用的教学方 法。虽然高度互动, 但因为讲授时教师面对全班, 而分组讨论时教师并不需要参与讨论, 对分课堂对班级 规模没有限制, 大班、小班教学都可应用。

\section{2 “对分课堂” 对教师和学生的要求}

理想的对分课堂不仅需要贯彻其基本理念, 还需要一整套教学措施来保障教学效果。无论是基本理念 还是具体措施, 在教学中都需要根据学校、教师、课程、学生的特点做一定的调整。

首先，分层教学会使教师转变教学观念，从而激发教师教学改革的积极性。更新教育观念是实施素质 教育的根本。在我们过去的教学过程中, 在教学方法上, 大多停留在 “满堂灌” “填鸭式” 的传统的教学 方法上, 滞留在老师怎么教我, 我怎么教学生的传统模式上。思想上残留着老师高高在上, 我讲你听, 我 讲你就应该会, 但是一提问, 学生往往一脸茫然……在注重日语学科特点, 开发学生潜能, 帮助学生如 何树立自信心上考虑的少, 学生作业、练习不会时只是批评, 如何帮助学生克服困难的少。这样的教学方 法, 导致各类测试都有相当数量的学生综合日语成绩不及格, 学习积极性严重不足。通过分层教学实验, 会让老师体会到自己肩上的压力, 感觉到传统教学方法的危害。

其次, 分层教学要求教师更加准确地把握课堂教学目标, 更加精确地选择教学内容, 更加恰当地运用 课堂教学方法, 在教学中既要面向全体学生, 又关心每一个体, 这促使教师要更认真地对待课堂教学的各 个环节, 认真分析学情, 刻苦钻研教材和教学大纲, 积极探索适合本层特点的课堂教学方法。

对分课堂教学还有一个要素, 即合适的教科书, 内容丰富、难度适中。这样, 教师进行框架性讲解后, 学生课后可以通过阅读教科书深入学习。教科书太容易, 学生学不到东西, 不能引发思考, 隔堂讨论就没 有内容; 教科书太难, 学生不能充分掌握, 隔堂讨论就缺乏深度。

此外, 分层教学会使学生愿意学习, 参与竞争, 从而使学习有较大进步。因为分层教学能充分关注学 
生的个性差异特点, 设立了恰当的教学目标, 选择了合适的教学内容, 保证了课堂教学内容最大限度地被 学生接受, 学生从中感受到成功的喜悦从而增强学习的信心, 开始尝试参与学习。因此, 分层教学存在着 竞争的氛围。这种氛围, 对那些要求上进的学生来说是一种动力, 将促使他们增强学习的主动性和积极性: 而对那些处于中下游的学生也是一种促进, 他们在自觉与不自觉之间都置身在这竞争的氛围中, 学习的效 果也得到了提高。

课堂是教学活动的主要场所, 课堂质量是教学质量高低的一个决定性因素, 在传统课堂上, 教师讲授 力求完整详尽, 学生没有发挥余地, 趋向被动接受。在对分课堂上, 老师有引导, 但并不穷尽内容, 留给 学生进一步主动探索的空间, 能够引发学生进行主动性学习。学生通过课堂上老师讲授获得基本框架, 理 解重点、难点, 大大降低了课后的学习难度, 在对分课堂中, 教学过程中的机械性成分降低, 指导性成分 提升, 教师角色从覆盖内容、灌输知识, 变为引导学生学习。学生在课下不学习很难参与讨论, 缺课也会 影响整 组讨论, 这些都会强化学生的团队合作意识。学生有机会同老师交流, 老师也可以随时参与讨论。 通过对分课堂生生、师生互动的幅度都大大提升。

对分课堂保留老师讲授这一传统教学的精华，保证了知识传递的系统性、准确性和有效性。对分课堂 提升了学生的课堂参与度, 老师不必为吸引学生注意去 “表演” , 而是回归到学生学习 “引导者” 的正确 定位上。老师虽然讲得少了, 但其地位和价值不但没有削弱, 反而得到进一步的提升, 更能赢得学生的尊 重。

\section{3 综合日语 “对分课堂” 的实践}

以新乡学院日语专业的综合日语课为例, 对分实践是基于自己的教学探索, 由于原本教学环节较多, 教师讲授与课堂讨论交流的时间比例都根据情况做了调整, 根据综合日语课程的特点设置作业的形式。采 取了教师讲授、课后作业、隔堂讨论三个步骤，对分课堂的主要流程清晰。

例如某次综合日语课布置了作业，让学生讨论课本其中一段包含的语法点，句子结构等，各个小组便 展开了讨论, 讨论后请一名代表在讲台上用日语发言, 小组每个成员轮流上台, 这样做, 可以使每一个学 生都有发言的机会, 也有听别人说的机会, 既有面对几个人发表自己见解的机会, 又有面对全班说话的机 会。有不擅长发言的同学, 开始站在讲台上对很多人发言, 紧张的完全不知所云, 本来私下交流, 日语发 音正确的学生, 也会由于紧张, 导致音调发不准确, 语法使用不当。这种情况, 初期会比较常见, 但是通 过多次课堂练习, 慢慢能够说的很好, 得到大家的一致赞赏, 并使学生对日语学习产生很大兴趣, 体会到 了成功的喜悦, 增强自信心。

小组内也相互交换各自发现的知识点, 通过小组合作, 提高了学生的学习效率, 巩固学习的知识, 并 由于一定程度的 “强迫使用日语表达” 这点上, 尝试用日语来交流, 为了表达自己的见解, 会更加主动地 思考、倾听、组织、灵活运用新旧知识, 使说出的语言更加准确、更全面; 讨论学习不仅加深了对知识的 理解, 也发展了学生的学习能力和语言表达能力; 从小组讨论这个方面来说对分课堂是一种非常好的教学 模式。

在传统课堂上, 教师讲授力求完整详尽, 学生没有发挥余地, 趋向被动接受。在对分课堂上, 老师有 引导, 但并不穷尽内容, 留给学生进一步主动探索的空间, 能够引发学生进行主动性学习。对分课堂提升 了学生的课堂参与度, 老师回归到学生学习的引导者的正确定位上。学生有机会同老师交流, 老师也可以 
随时参与讨论, 增多了师生互动交流的机会。

在本次综合日语对分课堂的教学过程中, 对应的考核方法强调过程性评价, 并关注不同的学习需求。 试点教学表明, 学生出勤率、作业完成情况、学习态度、成绩管理等方面都有显著提高, 对分课堂有效增 强了学生学习的主动性, 教学效果良好, 值得进一步尝试和推广。

\section{4 结束语}

在具体操作过程中, 可能我们还会受传统教学方法的影响, 无意中会忽视 “因人而异” 的价值而片面 地看待后进生的成绩, 一句不经意的批评指责之辞有可能会让他们丧失所有的学习热情。因此, 作为教育 工作者应该切记一条: 耐心、细心地呵护每一位学生, 多看到他们的长处, 多鼓励他们的进步。其他成功 的实验证明, 分层教学是面向全体学生, 提高日语听说读写四种技能, 全面调动学生积极性和创造性的一 种值得借鉴与深化的教学探索。

\section{5. 致谢}

新乡学院科技创新项目 “高校转型期英语专业二外日语教育的问题及对策”（编号：15SB33）

\section{Acknowledgements}

The Science and Technology Innovation Project of Xinxiang University_the Problems and Countermeasures of Japanese Education as the Second Language of English Majors（NO.15SB33） 参考文献:

[1] 席卫国. 论中国高校日语教育----中国特色日语教育研究 [M]. 陕西师范大学出版社， 2012，12.

[2] 曹大锋. 日语教育与教材创新研究---日语专业基础课程综合研究 [M]. 高等教育出版社, 2006， 10.

[3] 王尔馥. 网络环境下的高校对分课堂教学模式改革 [J]. 高师理科学刊, 2016, 5 .

[4] 余清臣, 徐苹. 当代课堂教学模式改革的实践内涵:一种反思的视角 [J]. 教育科学研究, 2014, 1 .

作者简介: 李万豫, 1972 年生, 女, 四川富顺 (籍贯), 讲师 (职称), 研究生 (学历), 研究方向: 日本文化

\section{References:}

[1] Xi Weiguo. Japanese Education in China's Colleges and Universities-Study on Japanese Education with Chinese characteristics [M].Shaanxi Normal University Press, 2012,12.

[2] Cao Dafeng. Research on Japanese Education and Textbook Innovation--Comprehensive study on Basic Courses of Japanese major [M]. Higher Education Press, 2006, 10.

[3] Wang Erfu. Network-based Reform on Teaching Mode for Half-and-half Class in Higher Education [J].College of Science Journal, 2016, 5.

[4] Yu Qingchen, Xu Ping. Practical Meaning for Current Reform on Teaching Mode: from a perspective of self-reflection [J].Educational Science Research,2014,1.

About the Author: Li Wanxiang, born in 1972, female, Sichuan Fushun (birthplace), Lecturer (title), post-graduate (education), research direction: Japanese culture. 